\title{
OSCILLATIONS IN SOLAR ATMOSPHERIC STRUCTURES
}

\author{
R. I. Kostik, E. Khomenko \\ Main Astronomical Observatoy, NASU, 27 Zabolotnogo Str., Kyiv, UA-03680, Ukraine
}

(Received February 2, 2004)

\begin{abstract}
The close proximity of the Sun makes it a uniquely observable star. The resolution of modern solar telescopes (both ground- and space-based) reaches $0 .^{\prime \prime} 1$, equivalent to $100 \mathrm{~km}$ on the solar disk, opens up new opportunities for studying the fine structure of the solar atmosphere, such as granulation, local oscillations, and the structure and variations of the magnetic field in quiet and active regions. The present paper gives an overview of the results of investigations of photospheric oscillations and their interaction with local structures (granulation and sunspots) in the Sun's atmosphere. We focus our discussion on the following topics: the effects of waves on spectral line profiles, the excitation mechanisms of oscillations, the hypothesis of local acoustic sources, the modulation of waves by the convective structure of the solar atmosphere, and observations and modeling of oscillations in sunspots. Finally, a short list of open questions is given.
\end{abstract}

Key words: the Sun, photosphere, oscillations, granulation, sunspots.

PACS number(s): 96.60.Ly, 96.60.Mz, 96.60.Qc, 95.30.Lz, 5.30.Qd

\section{THE EFFECT OF WAVES ON SPECTRAL LINE PROFILES}

The basic source of the information on the solar atmosphere is given by spectral lines. The asymmetry, broadening and shifts of spectral lines reveal the fine structure of the solar atmosphere. Interpretation of the solar spectrum at high spatial and temporal resolution demands realistic modeling of spectral lines without an any priori assumptions of local thermodynamic equilibrium (LTE) within the framework of inhomogeneous dynamic model atmospheres, taking granulation and waves into account. In this section we discuss the asymmetry and shifts of spectral lines caused by wave motions.

As early as 1948 Bierman [13] had proposed the idea that nonthermal broadening of spectral lines and their asymmetry can be caused by sound waves. Twenty years later, Eriksen \& Maltby [23] and Babij \& Altman [1] calculated the line absorption coefficient for a homogeneous atmosphere perturbed by progressive sound waves. The authors considered the case of velocity of absorbing particles, gas pressure, and temperature oscillating in phase, i. e. the process of sound wave propagation was assumed to be adiabatic. In this simplified case the line Doppler (i. e. velocity) shifts and pressure variations are connected linearly, the maximum red shift corresponding to the maximum of the pressure variations. The asymmetry of a spectral line in the presence of sound waves depends on the amplitude of density variations caused by pressure and velocity variations. In the weak line approximation (i. e. when the line is formed in a thin homogeneous layer) the line profile at each instant will be symmetric and will have a Gaussian shape, although its width, intensity, and shift will change in time. However, the line profile integrated over the wave period will not be symmetric. The value and sign of the asymmetry depend on the way of the wave propagation (adiabatic or isothermal) and on the atomic parameters of the line (excitation poten- tial, degree of ionization). Under equal conditions Fe II lines are less asymmetric than Fe I lines. The asymmetry is lower for adiabatic than for isothermal oscillations. The influence of radiative damping (i. e. the case when oscillations are neither adiabatic nor isothermal) and the height dependence oscillation properties were taken into account by Teplitskaja [84]. In this work the short-period $(2.5 \mathrm{sec})$ acoustic waves were considered. The calculated Fe I $\lambda 525.0 \mathrm{~nm}$ line profile appeared to be shifted and asymmetric even for a single moment in time. Kostik \& Orlova [50] have shown on the basis of observations and theoretical calculations (using the model of the wave propagation similar to [84]) that the asymmetry caused by waves with a period of 5 minutes constitutes only $20 \%$ from the observed value of asymmetry. However, comparison of results of the numerical modeling with observations was not carried out absolutely correctly since the observed asymmetry is caused not only by wave but also by convective motions.

From an observational point of view, the asymmetry of Fraunhofer lines caused by waves has been insufficiently investigated. In the numerous publications based on observations at high spatial and temporal resolution, the convective and wave motions are not as a rule separated. The average bisectors observed in granules and intergranular lanes filtered from the wave motions are given only in the papers by Hanslmeier et al. [36, 37]. Hanslmeier et al. $[36,37]$ found that waves can influence the shape of bisectors considerably. The waves have a stronger influence in intergranular lanes than in granules. However, these authors did not analyze the wave component of the bisectors.

A comparative analysis of the effect of waves and convection on the observed bisector shape and a comparison of the results of this analysis with theoretical calculations were performed recently by Kostik and Khomenko [2]. The fine structure of the Fe I $532.4 \mathrm{~nm}$ spectral line was studied on the basis of the high spatial and temporal res- 
olution observations of the quiet solar disc center. It was shown that the asymmetry of this line depends strongly on the nature of the velocity field. Asymmetry due to convective motions decreases from the line wings toward the line core. On the other hand, the asymmetry due to wave motions depends on time and increases toward the line core as a consequence of the increase of the amplitude of wave motions with height. The magnitude of the asymmetry due to waves is comparable to that due to convection. The distribution of the line shifts lies within $\pm 1 \mathrm{pm}$. The propagation of acoustic-gravity waves with a period of approximately 5 minutes in the 3D model of the solar atmosphere was considered. Kostik and Khomenko [2] solved the system of hydrodynamical equations for the case of a moving medium in order to model the wave propagation in granules and intergranular lanes. They found a rather good agreement between the observed and modeled bisectors due to wave motions. An example of Fe I $532.4 \mathrm{~nm}$ line bisectors is given in Fig. 1.
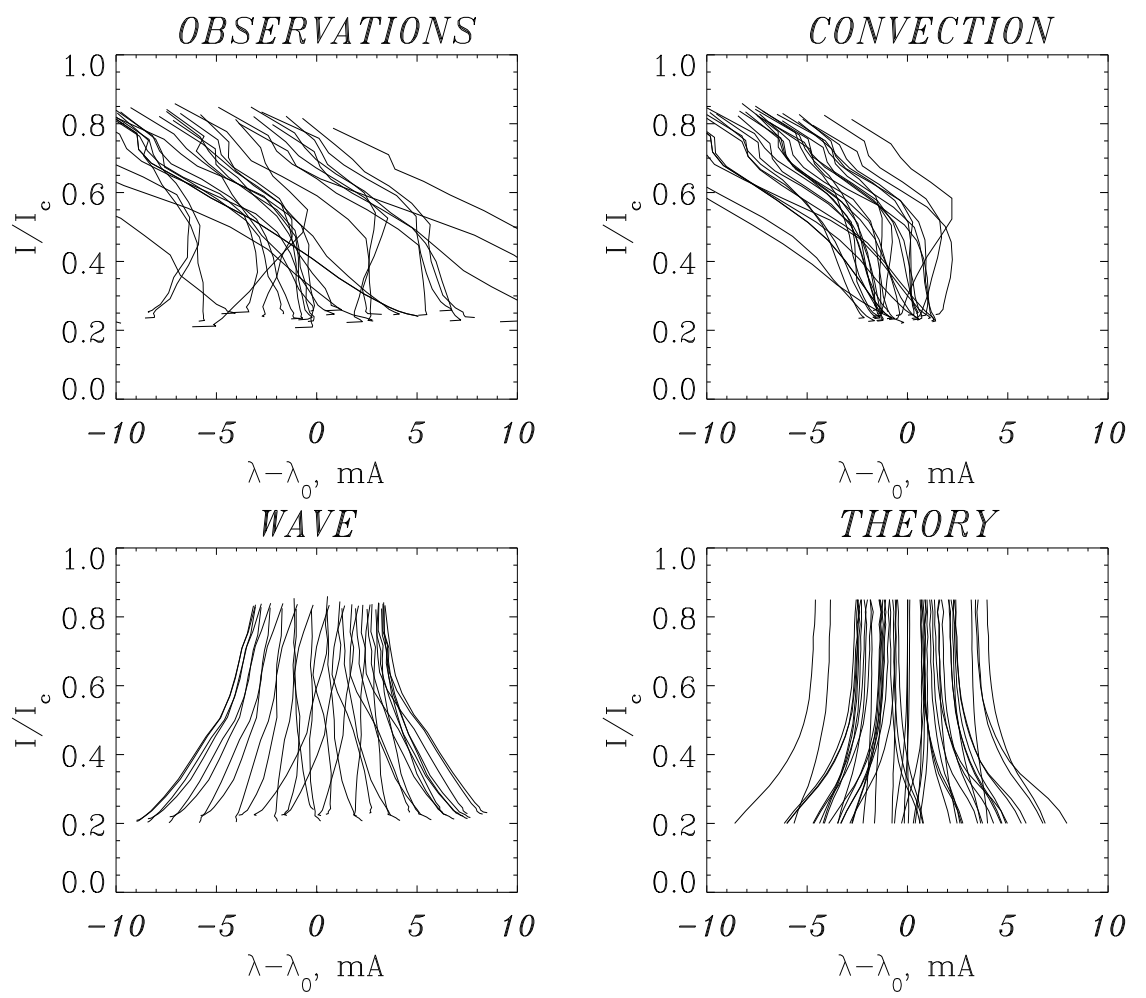

Fig. 1. Observed and calculated bisectors of the Fe I $532.4 \mathrm{~nm}$ line for the different time moments: (a) observed bisectors, (b) bisectors due to the convective component of the velocity field, (c) bisectors due to the wave component, (d) calculated bisectors for the wave component. Adapted from Kostik and Khomenko [2].

\section{EXCITATION OF OSCILLATIONS AND ACOUSTIC SOURCES}

The discovery of 5 -minute solar oscillations in the 1960s by Leighton and co-authors initiated a new field of solar physics - helioseismology. The oscillations got their name because the maximum of their power spectrum falls at the period of about 5 minutes. Depending on the value of the angular number $l$, the different modes of oscillations penetrate into different depths in the solar interior. The modes with lower values $(l=0,1,2)$ give information about the deepest layers down to the solar core, while modes with higher degree $l$ are trapped in the upper layers of the atmosphere and give information about inhomogenities in the top part of the convection zone and photosphere. Thus, by measuring the exact val- ues of frequencies of oscillations and solving an inverse problem of helioseismology it is possible to restore the values of the parameters of the atmosphere that produce the observed spectrum. Despite a vast amount of new information about the solar interior obtained by helioseismological methods, the question of the mechanisms of excitation of oscillations is still under intensive discussions.

\section{A. Excitation of oscillations by turbulent convection}

The initial assumption that rising granules are the direct source of waves $[25,57,74,80]$ found no experimental support $[28,60]$. The prevailing opinion for today is that solar $p$-modes obtain their energy from the stochastic 
processes of the turbulent convection just beneath the photosphere. This conclusion was derived for the first time in theoretical studies by Goldreich \& Keeley [30] and Goldreich \& Kumar [31]. To determine the acoustic energy flux generated by convection the authors have applied Lighthill's theory of excitation of sound waves [54] for the given spectrum of turbulent elements. The case of excitation of acoustic modes by fully or partly turbulent fluid was considered. The Kolmogoroff distribution of turbulent element velocities was assumed. Various types of turbulence were investigated, in particular, one of the types of forced turbulence, i. e. anisotropic turbulent convection, which takes place in a stratified atmosphere and is maintained by fluctuations in buoyancy force. An important result of Goldreich \& Kumar [31] is that in the case of turbulent convection the most highly excited modes have energies comparable to the thermal energies contained in a volume equal to that of an energy-bearing eddy, i. e. $E \sim m c_{s}^{2}$ (where $m$ is the mass associated with an energy-bearing eddy and $c_{s}$ is speed of sound). Since $m \sim \rho_{0}\left(v_{h} / \omega_{p}\right)^{3}$ ( $v_{h}$ is the turbulent velocity of an eddy of size $h$ and $\omega_{p}$ is frequency of oscillation of the mode), the equilibrium energy per mode is proportional to the velocity of the eddy to the third power $v_{h}^{3}$.

The above described method got a further development in the works of Kumar \& Goldreich [51], Goldreich \& Kumar [32], Balmforth [9], Bi \& Li [12], Goldreich et al. [33], Goode et al. [35]. Goldreich \& Kumar [32] performed theoretical calculations for the more realistic non-isothermal vertically stratified model atmosphere. The authors estimated the efficiency of the convective energy conversion into the wave energy for $p$-modes and propagating acoustic modes. The dimensionless efficiency appeared to be proportional to $M_{t}^{15 / 2}$, where $M_{t}$ is the value of the Mach number at the top part of the convection zone. Most of the energy going into the modes is emitted by eddies of size $h \sim M_{t}^{3 / 2} H_{t}$ (where $H_{t}$ is the value of the scale height at the top of the convection zone), at frequencies $\omega \sim \omega_{c}$ and wavelengths $\sim H_{t}$. The wave generation is concentrated in the top part of the convection zone since the turbulent Mach number peaks there. Bi \& Li [12] further improved the model of Goldreich \& Kumar [32] by considering the more realistic turbulent energy spectrum that follows the extended form of Kolmogoroff distribution. The Lighthill method was also applied by Nigam et al. [61,62] for an explanation of the opposite sense of asymmetry of the solar acoustic mode lines in velocity and intensity oscillation power spectra and also the high-frequency shift between velocity and intensity at and above the acoustic cutoff frequency observed by MDI/SOHO [22]. These authors have assumed that the granulation penetrating into the photosphere creates an additional noise at the same frequencies as $p$-modes. Since both granulation and oscillations have the same source, i. e. the turbulent convection, they are not independent and partly correlate between each other. This correlation produces the difference in the observed spectra of intensity and velocity. Nigam et al. $[61,62]$ found that the sign of the correlation is neg- ative, which suggests that there is a photospheric darkening prior to the occurrence of the localized acoustic event.

Recent numerical modeling of solar convection [29, $63,79]$ also confirms the model for stochastic excitation of oscillations. Three radial modes exist in simulations of Georgobiani et al. [29], Nordlund \& Stein [63], Stein \& Nordlund [79]. The properties of the profiles of these modes, in particular their asymmetry and phase shifts between oscillations of velocity and intensity, closely match those observed by MDI/SOHO [29]. Nordlund \& Stein [63], Stein \& Nordlund [79] came to the conclusion that the basic source of excitation of oscillations is stochastic non-adiabatic pressure fluctuations close to the surface. Since the pressure fluctuations are strongest in intergranular lanes and near the edges of granules, the oscillations are excited there more effectively.

Concluding what was said above, a model of the stochastic excitation of oscillations by convection successfully explains many properties of the observed spectrum of $p$-modes. However, it encounters some difficulties. Chaplin et al. [21] have shown, on the basis of the observed distribution of the low degree $l$ oscillation power, that certain features of the oscillations cannot be explained by the model of stochastic excitation by convection. These authors found an excess of the high-power events in comparison with what is predicted by the theory and suggested that the presence of this excess may reflect some other excitation mechanisms, for example external impacts. Foglizzo [27], based on the analysis the energy distribution of $p$-modes in the low $l$ range, also refers to the possibility of the existence of other sources of excitation of oscillations. Additional mechanisms that have been suggested are flares [47] and coronal mass ejections. The possible connection of magnetic activity on the Sun with the processes of excitation of oscillations was examined by Moretti et al. [58].

\section{B. Discrete acoustic sources}

A consequence of the model of stochastic excitation of oscillations by convection is the hypothesis of the existence of discrete sources of oscillations. Since the Mach number increases with increasing convective velocity and the emitted acoustic energy flux is proportional to $M_{t}^{15 / 2}$ [32], most of the emitted energy may come from rare localized events that are well separated from one another in space and time. The oscillations with the highest amplitudes should be generated above those areas of the solar surface where the convective velocities are highest. Such an assumption was made in 1991 by Brown [16], who suggested that the discrete sources of acoustic oscillations should exist and can be observed on the solar surface. If the reflection of upward-propagating waves is unimportant, almost all the acoustic energy from a buried source emerges nearby, within a limited area $A$ $\mathrm{Mm}^{2}$ and within a limited span of time $\tau$ sec. One expects that acoustic sources will be visible as discrete events provided that the wave fields from different sources do 
not overlap significantly, i. e. that the overlap likelihood, $L$, satisfies $L \equiv N A \tau<1$, where $N$ is the frequency of occurrence of a source, $\mathrm{Mm}^{-2} \mathrm{Sec}^{-1}$.

To estimate $N$, Brown [16] supposed that the highfrequency acoustic modes are excited by relatively highvelocity granular flows. Assuming that convective velocities below the photosphere follow a Gaussian distribution, the areas with maximum velocities should occupy the $f=0.005$ part of the surface. On the basis of this assumptions, Brown [16] found $N$ roughly to equal $2 \times 10^{-5} \mathrm{Mm}^{-2} \mathrm{sec}^{-1}$, i. e. one event per granular turnover time of $480 \mathrm{~s}$ within a circle of radius of $5 \mathrm{Mm}$. Employing a geometrical ray-tracing technique to follow the waves from their source, Brown [16] concluded that $90 \%$ of the energy is radiated within some $r=0.6 \mathrm{Mm}$ from the source. The geometrical configuration used in the ray tracing calculations is shown in Fig. 2. Taking this value of $r$ as a radius, it is possible to estimate the area occupied by a source on the surface as $A=1.1 \mathrm{Mm}^{2}$. The duration of the wave packet near the source $\tau$ is likely to be limited by a frequency bandpass within which oscillation data could be obtained. For a frequency interval $\omega$ $=7.5-5.5=2 \mathrm{mHz}, \tau=500$. Combining these results Brown [16] obtained $L \equiv N A \tau=1.3 \cdot 10^{-2}$, so that the inequality is completely satisfied. Thus, the conclusion was made that discrete sources of acoustic oscillations in space and time are likely to be observed on the Sun. However a spatial resolution much better than $1^{\prime \prime}$ is needed for their detection.

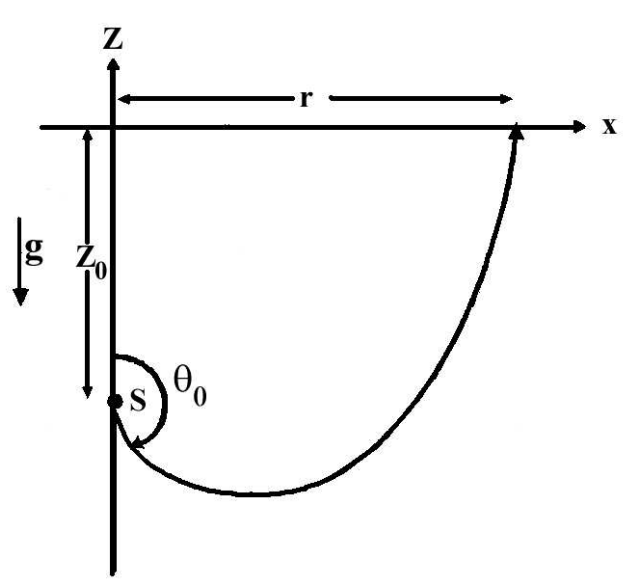

Fig. 2. The geometrical configuration used in the ray tracing calculations. A source $\mathrm{S}$ is buried at a distance $z_{0}$ beneath the reference surface at which wave motions are observed. A wave emerging, its initial propagation vector making an angle $\theta_{0}$ with the vertical, follows the indicated ray path, eventually emerging at radial distance $r$ from the source. Adapted from Brown [16].

The appearance of an acoustic event on the solar surface is characterized by a peak in the upward acoustic flux $\left(u \propto \frac{V^{2}}{\omega} \frac{\Delta \phi}{\Delta z}\right.$, where $V$ is velocity amplitude and $\Delta \phi / \Delta z$ is the observed phase gradient with the altitude of the event) followed by a peak in the velocity amplitude, which is then followed in about 5 minutes by a peak in the downward flux $[34,35,69]$. Due to the fast change of their phase with height, the waves propagating from the source are different from the trapped $p$-modes at the same frequencies. The phase of velocity oscillations for the $p$-modes changes insignificantly with height and this change is caused by the radiative damping of oscillations.

\section{Properties of granulation above an acoustic source}

What are the properties of granulation at the site of the appearance of the acoustic source? Goode et al. [34] associate the excitation of oscillations with isolated expansive events lasting about 5 minutes. The authors carried out numerical modeling of the excitation of oscillations by a rising piston located at some depth beneath the photosphere. The results of calculations were compared with high spatial and temporal resolution observations of the solar velocity field in the Fe I $\lambda 543.45 \mathrm{~nm}$ line [78]. The core of this line is formed high in the photosphere. Goode et al. [34] interpreted these observations as consistent with their numerical simulations and argued that the $5 \mathrm{~min}$ oscillations are excited not more than $200 \mathrm{~km}$ below the base of the photosphere where convective velocities are the highest. The best agreement with observations was achieved assuming that the sources of oscillations are discrete expanding elements of gas, and that these events are isolated in time. If this assumption is correct then, according to Brown [16], the maximum of the acoustic flux should be observed at the surface near the brightest granules.

However, in the majority of other theoretical investigations the appearance of an acoustic source on the surface was associated with a local darkening in photosphere (i. e. intergranular lane). According to theoretical investigations of Nigam \& Kosovichev [61], as already mentioned above, an explanation of the asymmetry of $p$ modes requires the assumption of correlated noise. This correlation is expected because the main source of noise is turbulent convection, which also drives the oscillations. The negative sign of this correlation obtained from the calculations of these authors supports the view that the local darkening of granulation should precede the appearance of an acoustic source. Numerical modeling of convection performed by Nordlund \& Stein [63], Stein \& Nordlund [79] shows that the oscillations are most effectively generated by non-adiabatic pressure fluctuations. The pressure fluctuations are strongest in intergranular lanes and near the edges of granules, and are also quite large in the most rapidly rising granules. However, in the latter case the absolute value of the fluctuations is almost twice as low as that in the intergranular lanes. Stein \& Nordlund [79] concluded that acoustic sources are located closer to the surface, basically in intergranular lanes and at the borders of granules. The conclusion about the effective wave excitation in intergranular lanes was also made by Rast [67] who proposed the mechanism of excitation of acoustic oscillations by localized cooling events and new downflow-plume formation at the solar surface. 


\section{Observed relations between oscillations and granulation}

Have acoustic sources been ever detected on the solar surface? There is a number of observational papers devoted to the question of the relations between granulation and local oscillations (e.g. [17, 24, 35, 40, 68, 69]). By local oscillations we mean oscillations that are locally excited in sub-photospheric layers and propagate away from the source. In this way the local oscillations differ from the $p$-modes that are trapped in the resonant cavity. As noticed by Hoekzema et al. [40], studying local oscillations in observations is complicated by the presence of global $p$-modes having higher power at the same frequencies. Local analysis (i. e. analysis of rather short time series of oscillations in each individual point at the surface of the Sun) using one-dimensional spectral data does not provide the statistics to estimate the importance of the observed events. On the other hand, Fourier transforms of large-area long-duration data sets reach statistical stability at the cost of mixing diverse regimes. Therefore, studying local phenomena requires short-duration Fourier analysis together with small-scale feature separation. One has to look for a compromise between the length of the time series and large temporal averaging masking the local properties of the oscillations. The results of investigations of local oscillations obtained by various authors are summarized below.

Brown et al. [17] investigated the solar velocity field at the frequencies of $5.5-5.7 \mathrm{mHz}$. They used observations in the Fe I $\lambda 557.6 \mathrm{~nm}$ line in an active region close to the solar disk center with a spatial resolution of about $1 .{ }^{\prime \prime} 5$. Brown et al. [17] found that a small fraction of the volume does indeed emit a disproportionate amount of the acoustic flux. The patches emitting most of the flux are located close to the regions with an enhanced magnetic field. It has been proposed that the increase in acoustic emission in a small part of surface (10\%) is related to the discrete acoustic sources predicted by Brown [16]. However, the role of the magnetic field is unclear.

Local oscillations were investigated by Restaino et al. [68] with the help of observations at high spatial $\left(0 .{ }^{\prime \prime} 67\right)$ and temporal (8.67 sec) resolution [78] in the Fe I $\lambda 543.4$ $\mathrm{nm}$ line. The core of this line is formed high in the photosphere allowing investigation of the height dependence of oscillation properties. The authors found acoustic events consisting of a pressure front followed by an oscillatory wake with a rapidly time-varying vertical phase gradient. The upward running waves were followed within 5 minutes by downward running waves. According to Restaino et al. [68] the observed oscillation wakes correspond to the acoustic events modeled by Goode et al. [34].

Rimmele et al. [69] made simultaneous high-resolution observations of the Sun's granulation and solar acoustic events at several heights in the photosphere. Their observations consist of broad-band images $\left(90^{\prime \prime} \times 90^{\prime \prime}\right)$ in white light close to the disk center accompanied by narrow-band filtergrams in the Fe I $\lambda 543.4 \mathrm{~nm}$ line. The authors applied a new method to study the statistical properties of acoustic events. It consists in computing the mean granulation intensity at the location of the largeflux acoustic events by locating events in space and time that reach amplitudes above the threshold and average intensity values in the pixels that have an acoustic flux above threshold. This procedure yields time variations in the mean acoustic flux (proportional to the amplitude of oscillations) and intensity of granulation averaged over the set of acoustic events. A similar method was used also by Goode et al. [35] and Strous et al. [83] in re-analysis of the same data. It was shown that local granulation begins to darken several minutes before the maximum in the flux, and that the granulation becomes darkest 2 minutes before the mechanical flux peaks. High-amplitude oscillations appear mainly above intergranular lanes. The latter contradicts the conclusions of Goode et al. [34] and Restaino et al. [68], but confirms the results of Nigam \& Kosovichev [61], Stein \& Nordlund [79] and Rast [67]. According to Goode et al. [35], Rimmele et al. [69], Strous et al. [83] the oscillations are most probably excited not by expanding granules penetrating into the photosphere [34] but rather are produced by rapid cooling during the formation of a new downflow in intergranular lanes, according to the theoretical calculations of Rast [67].

A detailed analysis of the spatial relations between granulation and 5-minute oscillations was carried out by Espagnet et al. [24] who used 57 minute time series of white light granulation images together with 16-minute time series of $\mathrm{NaID}_{2} \lambda 569.0 \mathrm{~nm}$ spectra. The granular and oscillatory components of the velocity field were separated with the help of a $k-\omega$ diagram. Analysis of histograms of granular velocities measured in pixels where amplitudes of oscillations exceeded a certain threshold value has shown that velocity oscillations tend to correspond to downflows, i. e. intergranular lanes. Besides, the $B-\omega$ diagrams (diagrams of the oscillatory power as a function of granulation brightness, $B$, and frequency, $\omega)$ revealed that power corresponding to 5-minute oscillations is much more concentrated in regions with the lowest intensities. Similar results were obtained for oscillations in the lower $(\sim 30 \mathrm{~km})$ and upper $(\sim 550$ $\mathrm{km}$ ) photosphere. Morphological analysis by Espagnet et al. [24] has shown that in most cases the oscillations arise directly in the center of intergranular lanes. Largeamplitude oscillations appear on the surface as new since their phase is distinct from that of weak oscillations at the same point. Espagnet et al. [24] interpreted their results as consistent with the model of stochastic excitation by turbulent convection [32].

Hoekzema et al. [38-40] proposed a new technique of Fourier-amplitude maps to study the relationships between different agents of the solar velocity field. The analysis was based on cospatial image sequences of the photosphere ( $G$-band filtergrams) and the overlying chromosphere (CaIIK filtergrams) at $430.8 \mathrm{~nm}$. For each spatial location, the partially overlapping time series of intensity variations were extracted. The Fourier transform of these series was performed and a 15minute average value of granulation contrast was assigned to each of the series. It appeared that the values of Fourier-amplitudes at $3 \mathrm{mHz}$ are nearly identical for var- 
ious classes of granulation contrast. Thus, the conclusion was made that "the photospheric 5-min oscillations are primarily global and rather insensitive to the local fine structure". At the same time the amplitudes of 3-minute oscillations appeared to be significantly above average in the dark intergranular lanes. According to Hoekzema et $a l$. [40] it means that without a special selection the variations of 5-minute period in the photosphere are dominated by global $p$-modes. In the chromosphere spectra are completely different from those at the photosphere. Both 3- and 5-minute oscillations have the largest amplitude above the darkest intergranular lanes. Hoekzema et al. [40] have drawn the conclusion that there is a local amplification of the amplitude of 3-minute oscillations above intergranular lanes in the photosphere and of 5-minute oscillations in the chromosphere. A qualitative scheme of the diffraction of waves on inhomogenities caused by granulation was offered to explain these results.

Some new insights into the physics of local oscillations are given by Kostik \& Shchukina [48], Kostik et al. [49], and Khomenko et al. [42, 44]. A 30-min time series of CCD spectrograms of solar granulation recorded with high spatial $\left(0 .{ }^{\prime \prime} 5\right)$ and temporal (9.3 s) resolution was used to study the links between 5-minute oscillations and granulation. The observed images contain the Fe I $532.4 \mathrm{~nm}$ spectral line with good height coverage from the low photosphere up to the temperature minimum region. Observational analysis led to the following results. (i) Strong oscillations are well separated temporally and spatially. (ii) Oscillations above granules and intergranular lanes occur with different periods. (iii) On average, the most energetic intensity oscillations occur above intergranular lanes; however, the situation is not so simple for the velocity oscillations; i.e., powerful oscillations may occur above granules as well. This situation is illustrated in Fig. 3. As can be seen, in the data of these authors the most energetic velocity oscillations occur above granules and lanes with maximum contrast, i.e. above the regions with maximum convective velocities. (iv) Velocity oscillations in the lower layers of the atmosphere lead oscillations in the upper layers in intergranular lanes. In granules the phase shift is nearly zero. The analysis of MDI/SOHO filtergrams performed in Khomenko [3] confirms the results of the groundbased observations of Kostik \& Shchukina [48], Kostik et al. [49], and Khomenko et al. [42,44].
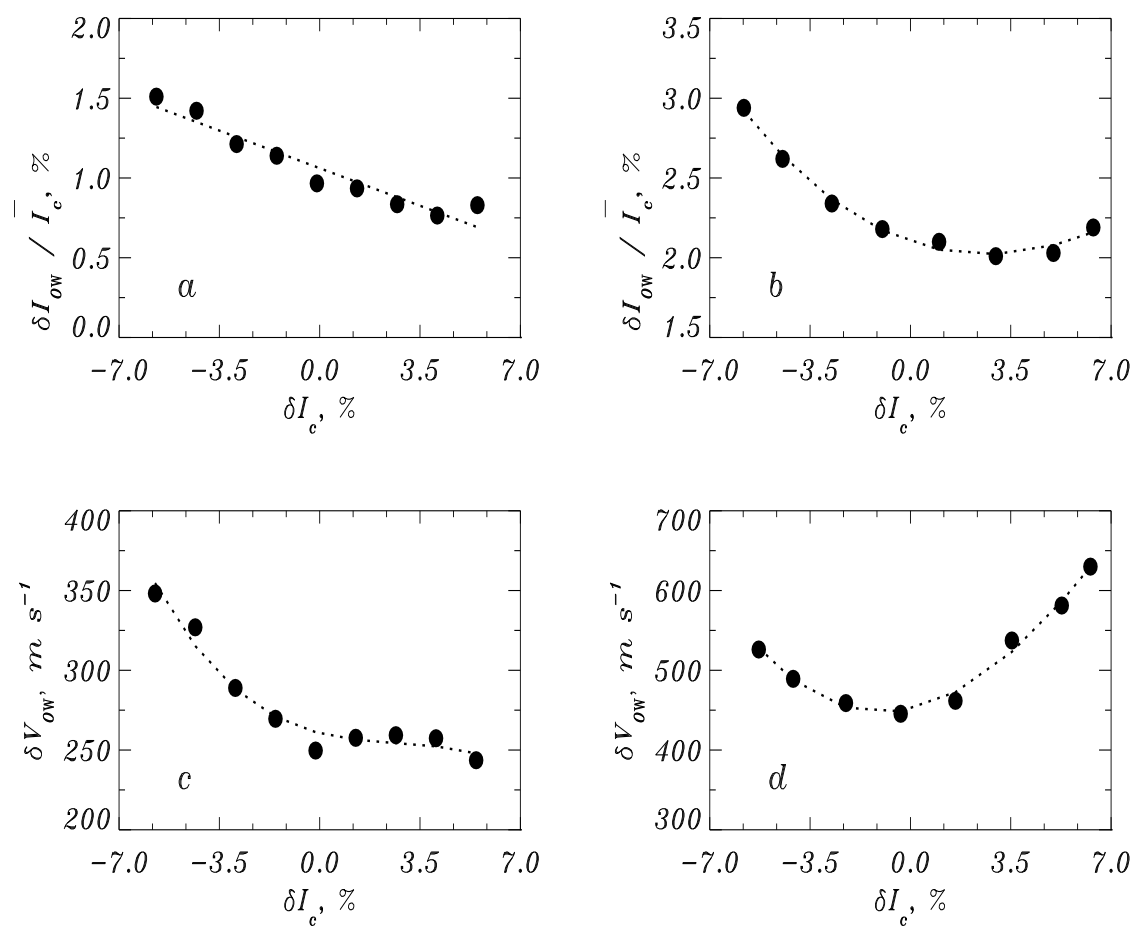

Fig. 3. (a) and (b): amplitudes of the line core intensity oscillations $\delta I_{0 W} / \bar{I}_{c}$ as a function of continuum contrast $\delta I_{c}=\left(I_{c}-\bar{I}_{c}\right) / \bar{I}_{c}$. (c) and (d): same for velocity oscillations $\delta V_{0 W}$. Amplitudes in panels (a) and (c) are measured as extreme values in each oscillating element and then averaged over contrast intervals. Amplitudes in panels (b) and (d) are measured as maximum values in each oscillating element. Adapted from Khomenko et al. [44]. 
Summarizing the results of this paragraph we see that 5-minute oscillations are indeed observed to be different above granules and intergranular lanes. Observations with high spatial resolution by Espagnet et al. [24], Goode et al. [35], Rimmele et al. [69] show that the amplitudes of velocity and intensity oscillations are, on average, larger above intergranules. It was revealed also that waves in intergranular lanes are characterized by a stronger change of their phase with height. These data were interpreted as a confirmation of the hypothesis that discrete acoustic sources can be observed on the solar surface, and that these sources are located in the dark intergranular lanes [24,35,69]. At the same time Kiefer et al. [45], Kostik \& Shchukina [48], Kostik et al. [49], Khomenko et al. [44] (see Sec. III below for details) found that powerful velocity oscillations may also arise above bright granules (see also [68]), and the results of Hoekzema et al. [38] show that the amplitudes of the photospheric 5-minute oscillations above granules and intergranules are identical. The above cited papers contradict the results of Espagnet et al. [24], Goode et al. [35], Rimmele et al. [69]. We thus conclude that, despite considerable efforts by different researchers, the issue of the links between 5-minute oscillations and granulation remains open. In particular, it is not clear whether the different behavior of oscillations above granules and intergranules can be explained only by the unequal efficiency of their generation in these structures or whether there are other reasons for this difference.

\section{MODULATION OF OSCILLATIONS BY THE CONVECTIVE STRUCTURE OF THE ATMOSPHERE}

It seems natural that the properties of oscillations should depend on the atmosphere in which they propagate, i. e. in rather hot rising granules or in cool descending intergranules. The assumption that the modulation of waves by inhomogenities in the solar atmosphere significantly influences their observed properties was made in the papers by Kiefer et al. [45], Murawski \& Penilovski [59], Rosenthal et al. [70], Stix [81], Stix \& Zhugzhda [82], Zhugzhda [87]. There are not many theoretical investigations devoted to this problem.

Zhugzhda [87] suggested a theoretical model of acoustic oscillations in a one-dimensional periodic environment composed of a sequence of vertical channels with cold downflowing and hot upflowing material. Vertical component of the convective velocity and sound speed in this model depend on the horizontal coordinate, $x$, as:

$$
\begin{aligned}
& V(x)=\hat{c}_{s}\left(V_{m}+2 \Delta \cos \pi x / d\right), \\
& c_{s}^{2}(x)=\hat{c}_{s}^{2}(1+2 \delta \cos \pi x / d)
\end{aligned}
$$

where $2 d$ is the horizontal period of the model, $\Delta$ and $\delta$ are the amplitudes of variations of convective velocity and sound speed (caused by temperatures variations), $V_{m}$ and $\hat{c}_{s}$ are average values of the latter quantities, and
$V_{m}$ and $\Delta$ are measured in terms of $\hat{c}_{s}$. These equations define a one-dimensional model for stationary convection (or turbulent convection that is slow relative to the acoustic timescale). The theoretical calculations of the author were based on the theory of acoustic waves in a crystal lattice and some of the concepts of the solid state physics were generalized to the problem of acoustic waves in a convective atmosphere. The model for oscillations, however, does not take gravity into account. Thus, in case of the solar atmosphere the model is correct only for acoustic oscillations with the frequencies higher than 5-6 $\mathrm{mHz}$. Zhugzhda [87] derived the wave equation governing acoustic waves in a structured periodic atmosphere. The coefficients of this equation are dependent on the horizontal coordinate, $x$. The solution of this equation has the following form:

$$
y=\exp i\left(\omega t-k_{z} z+k_{x} x\right) \sum_{-\infty}^{\infty} C_{2 m} \exp \left(\frac{i m \pi x}{d}\right)
$$

where $y(x)=\nabla \mathbf{v} /\left(\omega-k_{z} V(x)\right)$ and $\mathbf{v}$ is the wave velocity. Thus, $\mathbf{v}$ depends on the parameters of the convective structure. The coefficients $C_{2 m}$ can be found from recurrence relations after the substitution of the solution into the wave equation and depend on the parameters $d, \Delta$, and $\delta$ of the model. It was shown that three wave modes exist in such a periodic atmosphere: acoustic waves, vibrational waves, and turbulent sound. All of them are different from the usual acoustic waves in a uniform atmosphere. Based on the model of Zhugzhda [87], Stix \& Zhugzhda [82] calculated the corrections to the eigenfrequencies of the $p$-modes arising from the sound speed and velocity inhomogeneity in the convection zone. The absolute values of the frequency shifts are significant $\left(\sim 5 \times 10^{-3} \mathrm{mHz}\right)$ and allow us to reduce the difference between the observed frequencies of the oscillations and those calculated in a standard solar model atmosphere. Thus, the influence of the inhomogenities of the atmosphere caused by convection should not be considered insignificant. Stix \& Zhugzhda [82] found that most of the effect arises from the velocity fluctuations, while only a small fraction comes from the sound speed fluctuations. One of the reasons for this difference is that $\delta$ in a standard model of solar convection decreases with depth much faster than $\Delta$.

Recently, a detailed theoretical investigation of the propagation of waves in a non-uniform solar atmosphere was carried out by Kiefer et al. [45]. The authors considered, on the one hand, the modulation of running sound waves in the outer convection zone, and, on the other hand, the impact of local acoustic sources on the observed properties of oscillations. A direct comparison was made of theoretical calculations with the behavior of photospheric running sound waves extracted from observational velocity data at two heights in the photosphere. Applying the model of the wave modulation of Stix \& Zhugzhda [82] (see Eq. 2), Kiefer et al. [45] has shown that the amplitude of vertically propagating waves, $v_{z}$, in hot upflowing and cold downflowing channels is not 
the same for waves in the frequency range $3-9 \mathrm{mHz}$. The amplitude, $v_{z}$, of the waves with frequencies $5-7.5 \mathrm{mHz}$ is larger in bright channels, however, the ratio between the amplitudes in bright and dark channels decreases with increasing frequency. At higher frequencies the amplitude of oscillations becomes larger in dark channels. For the considered granular structure this transition occurs at frequency near $7 \mathrm{mHz}$. The ratio of the amplitudes is considerable and varies within $2.5-1$, depending on the frequency and the parameters of the model (see Fig. 4, where the amplitudes of waves in bright and dark channels are given). Since the temperature structure of granulation persists as overshooting up to $100 \mathrm{~km}$ and the velocity structure persists over several hundred $\mathrm{km}$ in the photosphere, the degree of modulation of waves (according to [45]) should increase or, at least, should not decrease with height. The modulation should be more pronounced for waves with a lower horizontal wave number, $k_{x}$, since such waves always propagate within the limits of the same bright or dark channel.

$\nu=7.3 \mathrm{mHz}$

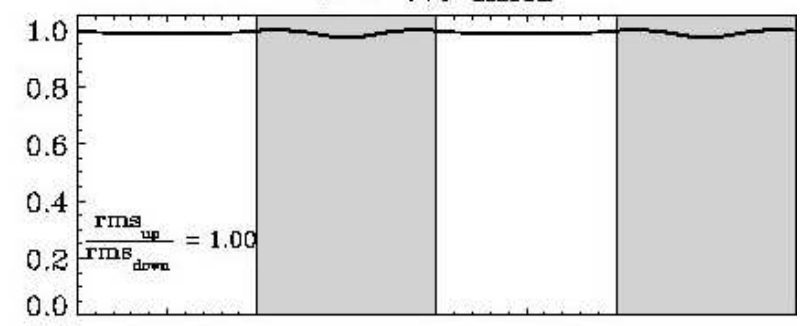

$\nu=6.2 \mathrm{mHz}$
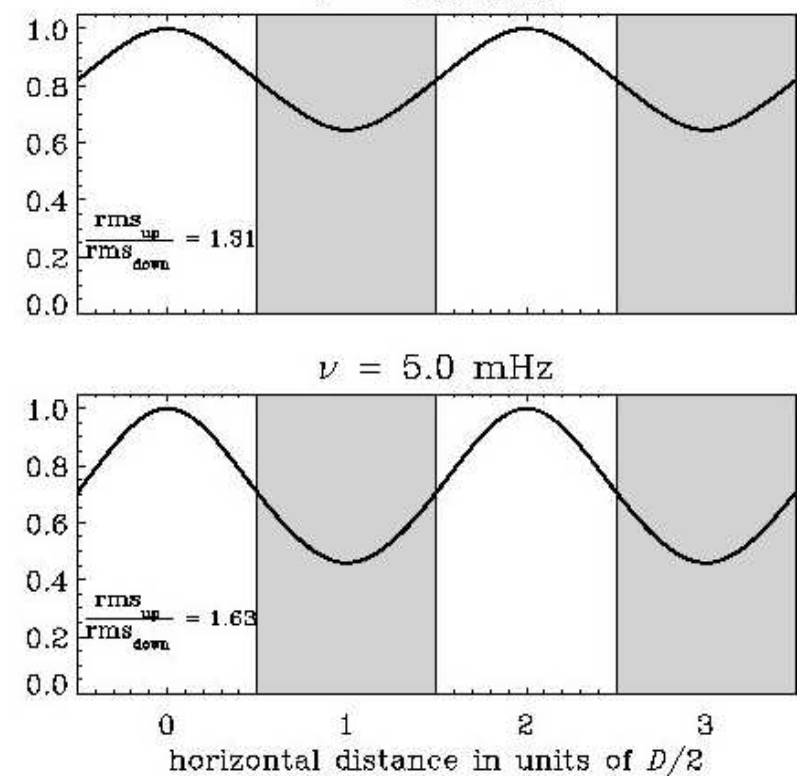

Fig. 4. Horizontal course of the normalized amplitude of the vertical velocity, (thick curves) from the $2 \mathrm{D}$ harmonic model of Stix \& Zhugzhda (1998) for three frequencies in the range of photospheric vertically propagating sound waves. $D$ is the horizontal period of the inhomogeneities. Shaded areas indicate the region of cool, downward flowing material. The ratio of the rms-amplitudes of between regions with upstream and regions with downstream is given in each panel. Taken from Kiefer et al. [45].
Kiefer et al. [45] modeled the wave field from an acoustic source at frequencies above the acoustic cut-off and assumed that the acoustic sources are isolated one from another, i. e. their velocity field does not significantly interfere, and that the wave field from the source is axisymmetric with respect to the vertical axis. The velocity potential of the wave field depends on the spherical polar angle, $\theta$, and the distances, $s$, from the source as $\psi_{l, n} \propto P_{l}(\theta) s^{-n}$, where $l$ and $n$ are integer numbers, and $P_{l}(\theta)$ is Legendre polynomial of degree $l$. The above dependence means that the amplitude, $v_{z}$, decreases with increasing distance from the source both in the vertical and in horizontal directions. The acoustic sources in the model were located in the brightest granules and the darkest intergranular lanes.

To check the above described models Kiefer et al. [45] used time series $(\sim 2$ hours) of images of a quiet area close to the disk center in white light (of size $65^{\prime \prime} \times 43^{\prime \prime}$ ) accompanied by Doppler velocities at heights of 50 and $250 \mathrm{~km}$ in the photosphere obtained from the profiles of the Fe I $\lambda 709.04 \mathrm{~nm}$ line registered quasi-simultaneously. The variations of these quantities caused by granulation and running sound waves (with frequencies above $5 \mathrm{mHz}$ ) were separated based on the $k-\omega$ diagram. Granulation intensity was split into ten contrast classes. The amplitude of velocity oscillations was determined separately for each class. The dependence of the amplitudes on contrast appeared to be different for different frequencies, horizontal wave numbers, and heights in the atmosphere. Comparison of theoretical calculations with observations shows that at a height of $50 \mathrm{~km}$ the model of acoustic sources located in dark intergranular regions gives a very satisfying description of observations for those classes with the lowest granular intensity (i. e. for intergranules). However, at a height of $250 \mathrm{~km}$ there is a systematic frequency-dependent deviation between the observed and theoretical curves in the sense that observations show larger power of oscillations above bright granules. The observed amplitudes of oscillations at the height $250 \mathrm{~km}$ are larger above granules than above intergranules, however the difference in amplitudes decreases with increasing frequency. The observed distribution of amplitudes for lower-frequency oscillations $(5-6 \mathrm{mHz})$ at a height of $250 \mathrm{~km}$ corresponds rather well with that expected in the case of the wave modulation. At the same time, the model with an acoustic source gives better agreement between theory and observations for more high-frequency oscillations $(6-8 \mathrm{mHz})$. Kiefer et al. [45] came to the conclusion that the influence of the acoustic sources is high in the data from lower heights and the impact of wave modulation is stronger in the data from lower heights. Altogether, the distribution of the velocity amplitudes with contrast is possible to explain by taking into account only the joint action of the local acoustic sources and the wave modulation by the atmosphere.

Simultaneously with Kiefer et al. [45] the model of Stix \& Zhugzhda [82] was applied by Stix [81] for a detailed investigation of variations of amplitude and a phase of acoustic oscillations due to the wave modulation in the 
solar convection zone. The velocity oscillations in the periodic model atmosphere were computed applying Eq. 2. It was shown that a vertically propagating wave experiences only the modulation of the amplitude. In the case $V(x)=0$, i. e. where the atmosphere is structured only in temperature, the amplitudes of the waves propagating upward and downward are identical. The amplitude of low-frequency oscillations appeared to be larger in bright channels while the amplitudes of high-frequency oscillations are larger in the dark ones, in agreement with Kiefer et al. [45]. The transition between these two regimes occurs at the frequency for which the vertical wavelength of oscillations is equal to the horizontal period of the inhomogeneity of the atmosphere, i. e. $k_{z}=\pi / d$. In the case $c(x)=$ const, i. e. when only velocity inhomogenities are present in the atmosphere, the upward propagating wave behaves similarly to the case of temperature inhomogenities. The downward propagating wave behaves in the opposite way; namely, its amplitude in the downflowing channels is approximately equal to the amplitude of the upward propagating wave in the upflowing channels. The result of the combined action of the temperature and velocity inhomogenities depends on the relation between their amplitudes $\Delta$ and $\delta$. Since the modulation has the same sign for the upward propagating waves and has the opposite sing for the downward propagating waves, it is less pronounced in the latter case at the combined action of both agents. Waves propagating at an angle to the vertical experience the modulation of both the amplitude and the phase. The phase of the upward propagating wave is nearly constant in dark channels and advances in bright channels. The absolute value of the modulation decreases with frequency, reaching a minimum for the frequency of the transition and then increases again. Taking a sound speed value of $\hat{c}=10 \mathrm{~km}$ and a characteristic granulation size of $2 d=1280 \mathrm{~km}$, Stix [81] has estimated the frequency of the transition to be about 5 $\mathrm{mHz}$. This value $(7 \mathrm{mHz})$ is lower than that given by Kiefer et al. [45]. The results of the modeling by Kiefer et al. $[45,81]$ are in qualitative agreement with the theoretical research of Murawski \& Penelovski [59]. In the latter paper the influence of the random flow occurring in the convection zone on frequencies of acoustic oscillations was considered. The numerical results reveal that waves can be both amplified and dampened by the random flow depending on its characteristic horizontal size. Granules of sizes about $0.2 \mathrm{Mm}$ cause an increase in the wave amplitude, while granules of larger sizes $(2 \mathrm{Mm})$ cause the opposite effect.

A different approach was adapted by Khomenko et al. [44] and Khomenko [3], who reconsidered a standard theory of acoustic-gravity oscillations making an attempt to generalize it for the case of oscillations in moving media. To this aim oscillations were introduced into the numerical 3D convective model of the solar atmosphere [5]. To take into account the change of the granular velocity and temperature with height, the atmosphere was split into a set of isothermal layers with constant values of these parameters and the solution of the set of hydrodynamical equations was computed separately in each of the layers. The effects of the wave reflection from the boundaries of these isothermal layers were considered. The perturbed model atmosphere was used to synthesize the profiles of the Fe I $532.4 \mathrm{~nm}$ and Ni I $676.8 \mathrm{~nm}$ lines (used in observations) and to make a direct comparison of the observed and theoretical results. The oscillations with a period of around 5 minutes were considered. The model for oscillations that takes granular velocities and wave reflection into account allows us to explain qualitatively the observed dependencies without any assumption about the different rate of excitation of oscillations in granules and lanes. Namely, the numerically computed 5-minute velocity oscillations appeared to have larger amplitudes above intergranular lanes at all heights in the photosphere (see Fig. 5), in agreement with observations. The amplitudes of the observed intensity oscillations are larger above intergranules. If the reflection of waves is not taken into account, the theoretical intensity amplitudes agree with the observations only at heights above 200-250 km (compare curves at panels (a) and (b) of Fig. 5). The account for wave reflection leads to a decrease in the intensity oscillatory amplitude above granules in deep layers and thus a better correspondence to observations is achieved.

Altogether, theoretical investigations show that the modulation of waves by the convective structure of the atmosphere cannot be neglected. Accounting for the convective structure allows to explain qualitatively the difference between the observed and calculated in the standard solar model atmosphere frequencies of oscillations. The difference in the amplitudes of oscillations in bright and dark channels is significant and can be detected in observations. The modulation of waves by the atmosphere can be an additional or alternative mechanism in the interpretation of the difference in properties of oscillations above granules and intergranular lanes. The modeling performed by Kiefer et al. $[45,59,81,82,87]$ is applied only for high-frequency $(>5-6 \mathrm{mHz})$ acoustic oscillations in the solar atmosphere. It would be interesting to consider the influence of gravity (i. e. to consider oscillations in a vertically stratified atmosphere). This will enable us to investigate the modulation of relatively lowfrequency 5 -minute oscillations. The numerical experiment of Khomenko et al. [44] and Khomenko [3] shows that the effects of temperature and, in particular, velocity structuring of the atmosphere on the acoustic-gravity oscillations at $3 \mathrm{mHz}$ are potentially significant and are able to account for the observed difference in the oscillatory amplitudes and phases above granules and lanes. However, a more strict solution of the problem is desirable in order to reach a final conclusion. 

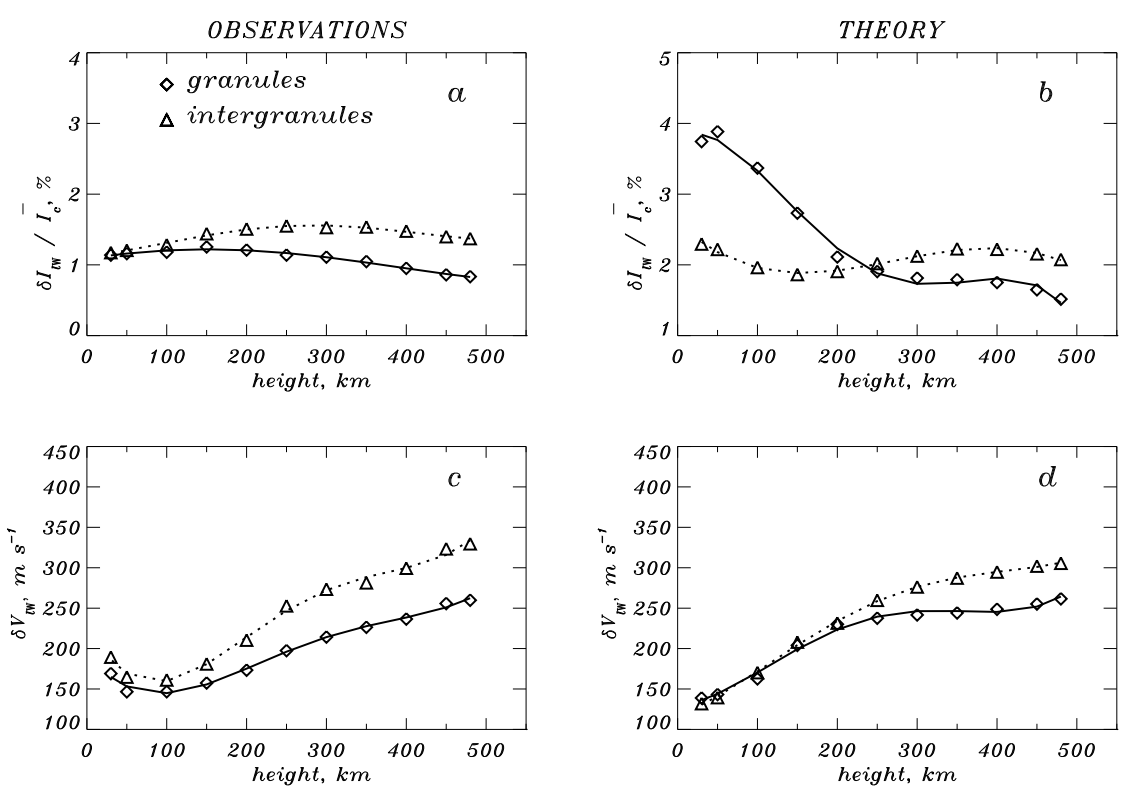

Fig. 5. (a) and (b): Height dependence of the intensity oscillatory amplitude $\delta I_{l W} / \bar{I}_{c}$ above granules and intergranular lanes. (c) and (d): Same for velocity oscillatory amplitude. Amplitudes are measured at different heights and then averaged separately for oscillations above granules and lanes. Taken from Khomenko et al. [44].

\section{OSCILLATIONS IN SUNSPOTS}

Sunspots show a variety of oscillatory phenomena. We refer the reader to the recent overviews of the structure and physics of sunspots given by Staude [77], Bogdan [14], and Solanki [76]. Oscillations in sunspots are an actively debated topic for several reasons. On the one hand, the helioseismology of sunspots has as an objective the determination of their subsurface structure using information about oscillations [46]. These deep layers are impossible to investigate with spectroscopical methods. On the other hand, oscillations in active regions are believed to play a role in the heating of the upper solar atmosphere, chromosphere, and corona.

According to the review by Staude [77] there are three bands of oscillations in the sunspot umbra with resonant periods around $2-3 \mathrm{~min}, 5 \mathrm{~min}$ and $>20 \mathrm{~min}$. Bogdan [14] divides sunspot oscillations into three categories: 5-min umbral-photospheric oscillations, 3-min umbral-chromospheric oscillations, and running penumbral waves. The different classes of oscillations are possibly produced by different physical mechanisms. Each of these phenomena could be the subject of a separate review. Below we will focus the discussion of 5-minute photospheric oscillations in sunspots umbrae.

\section{A. Oscillating quantities and relations between them}

Oscillations in sunspots were discovered more than 25 years ago (almost 10 years later than oscillations in quiet regions, owing to the lower amplitudes of the former). Velocity (i. e. Doppler shifts) and intensity (i. e. thermodynamic parameters) oscillations are shown in observa- tions in the spectral lines formed at various levels in the atmosphere of sunspots. The amplitudes of the 5-minute photospheric oscillations decrease with height until their complete disappearance in the upper chromosphere and corona. Attempts to construct a diagnostic $k-\omega$ diagram for these oscillations are not numerous $[4,66]$. The spectrum of the velocity and intensity oscillations observed in sunspots appeared to be similar to that in the quiet areas $[4,14,66,77]$; however, significant power is present only at low frequencies $k$ and $\omega$. Powerful oscillations are limited in the range of the horizontal phase speeds of about $25 \mathrm{~km} \mathrm{~s}^{-1}$, which is close to the Alfven speed in the photosphere. The 5-minute umbral oscillations are coherent over a significant fraction of the sunspot umbra and extend into a portion of the neighboring penumbra. The areas with enhanced power of oscillations isolated in space are probably caused by the interference of many modes, similar to the situation in the quiet Sun [14].

Although the oscillations of velocity and intensity are relatively well studied, very little is known about magnetic field oscillations in sunspots. Temporal variations of the magnetic field in sunspots were detected for the first time in observations by Horn et al. [41]. It is unclear whether the observed variations are caused by intrinsic oscillations of the magnetic field. The detection of the intrinsic oscillations is still a matter of debate and the results by different authors are contradictory. The existing theoretical models predict the amplitudes of oscillations to be very low, about a few G (see below Sec. IV C [55]). Measurements with such precision can hardly be achieved with the existing level of observational techniques.

The data on the spatial distribution of the power of magnetic field oscillations and their relations with the 
velocity oscillations are contradictory. The MHD/SOHO observations hint that power of the magnetic field oscillations is not distributed randomly over active regions. The decrease in the frequency of oscillations with decreasing magnetic flux is reported by Norton et al. [64] from an analysis of MDI magnetograms. For the lower oscillatory frequencies most of the magnetogram power appears in lower flux pixels $(100-300 \mathrm{G})$ in a ring of filamentary structure outside the sunspot. For the 5-min range the prefered locations are pixels with a magnetic flux of $600-800 \mathrm{G}$. The 3 -min band oscillations are observed in 1300-1500 G pixels. At the same time, no difference in the power pattern for any frequency ranges was found in the spectral observations by Balthasar [10] (see also [88]). Balthasar [10] and Zhugzhda et al. [88] used time series of images of active areas obtained by scanning with the Fabry-Perot interferometer (FPI) of the magneto-sensitive line Fe I $\lambda 584.3 \mathrm{~nm}\left(g_{\text {eff }}=2.5\right)$. The value of the magnetic field strength, $B$, was determined by comparison of the observed line profiles with those computed theoretically for a set of $B$ values. The FPI observations of Balthasar [10] and Zhugzhda et al. [88] revealed that temporal variations of magnetic field occur at the umbra-penumbra transitions and in the central parts of some sunspots and pores. The above results suggest that the patches with the enhanced magnetic field power are highly localized in space.

Both FPI and MDI data show that the patches with the amplified oscillations of the magnetic field are considerably smaller than in case of velocity oscillations. FPI observations demonstrate a high correlation between 5minute and, in particular, 3-minute velocity oscillations and oscillations of the longitudinal (parallel to the line of sight) component of the magnetic field $[41,88]$. The maximum power of the velocity and magnetic field oscillations corresponds to those areas in the umbra where the magnetic field lines are parallel to the line of sight (LOS). As the sunspot observed by Horn et al. [41] and Zhugzhda et al. [88] was located outside the solar disk center, those are the regions close to the border of umbra/penumbra. The patches with the powerful 5-minute magnetic field oscillations in the MDI observations are also located mainly in the penumbra [64]. Nevertheless, no coherence is found between spatial distribution of variations of the magnetic field and those of Doppler velocity and intensity (see also results from MDI data by [73]). A detailed analysis of the magnetic field oscillations in sunspot penumbrae based on spectropolarimetric observations of the full Stokes vector of the infrared Fe I lines at $1.56 \mu \mathrm{m}$ has been performed recently by Bellot $\mathrm{Ru}-$ bio et al. [8]. Temporal variations of the magnetic field, velocity and thermodynamic parameters were inferred from observations by inversion method. The spectropolarimetric observations [8] show that the amplitude of the 5 -minute magnetic field oscillations is at its maximum in the center of the umbra and decreases toward the border of the umbra/penumbra. This does not agree with the results of Horn et al. [41], Norton et al. [64] and Zhugzhda et al. [88]. The amplitude of velocity oscillations is the lowest in the center on the umbra and increases towards the penumbra, in agreement with Horn et al. [41] and Lites et al. [55].

Estimates of the amplitudes of the magnetic field oscillations and phase relations with velocity oscillations are very uncertain and vary from one measurement to another. Lites et al. [55], based on the inversion of the full Stokes vector of the Fe I $630.15 \mathrm{~nm}$ and Fe I 630.25 $\mathrm{nm}$ lines, reported an upper limit of the amplitude of oscillations of about $4 \mathrm{G}$ and considered them rather to be produced by instrumental effects. Rüedi et al. [73] found amplitudes of about $6 \mathrm{G}$. At the same time Balthasar [11] obtained substantially larger amplitudes of up to $50 \mathrm{G}$ in individual patches of enhanced oscillations. In the observations by Bellot Rubio et al. [8] the maximum of the magnetic field power occurred at $3.75 \mathrm{mHz}$ with the amplitude decreasing with height from $11 \mathrm{G}$ at $\log \tau_{5}=0$ to $8 \mathrm{G}$ at $\log \tau_{5}=-1$.

Phase relations are important for the diagnostic of the type of observed oscillatory phenomena. Most observations give values of about 90 degrees with upward velocity leading the magnetic field, with the positive velocity direction towards the center of the Sun $[8,11,64,73]$. Nevertheless, in several cases the correlation between velocity and magnetic field oscillations was found; i. e. the phase shift between them is equal to zero [41,55]. Other atmospheric parameters such as temperature and magnetic field inclination do not show an oscillatory behavior $[8,55]$.

The contradictions between the results derived by different authors are most probably the consequences of the differences in observational procedures, their sensitivity to the magnetic field, size of the area covered by observations. It is important to keep in mind whether the full magnetic field vector is determined (as in the case of the inversion of Stokes profiles of spectral lines) or just its longitudinal component (as in the case of magnetograms). Apart from intrinsic magnetic field oscillations, there are a number of effects that can cause observed variations of the magnetic field. These are instrumental effects, variations in the atmospheric seeing (in the case of ground-based observations), and oscillations of the velocity and intensity of spectral lines. One of the most important effects is that of the time variations of opacity of the solar atmosphere (we shall consider this in more detail in the next paragraph). The MDI data are not affected by seeing oscillations. However, as MDI determines the magnetic field by means of filtergrams, the time variations of the intensity and velocity of the Ni I 676.8 line can cause false oscillations of the magnetic field [72]. The results of the measurements of the magnetic field in non-simultaneous registration of the line profile (as in the case of MDI and FPI) were questioned in the paper by Settele et al. [75]. The authors have shown that the variations of the magnetic field measured by MDI and FPI are strongly contaminated by the velocity oscillations. The amplitude of false magnetic field oscillations caused by this effect depends linearly on the amplitude and frequency of the velocity oscillations and on the magnetic field strength in sunspot. In case of FPI, the amplitude of the false oscillations is of the same order 
of magnitude as in the observations $[11,41]$ while in the case of MDI it is a little lower than in the observations. The $1.56 \mu \mathrm{m}$ Fe I lines used by Bellot Rubio et al. [8] are probably the best tool for studying such weak effects as magnetic field oscillations because of their great magnetic sensitivity. The SIR inversion [71] applied by Bellot Rubio et al. [8] allows the separation of the influence of the different atmospheric parameters and provides reliable results on the magnetic field vector as a function of time and optical depth $\tau_{5000}$. Apparently, the results of the measurement of the magnetic field oscillations by Bellot Rubio et al. [8] are the most reliable in published literature today.

\section{B. Opacity oscillations in the solar atmosphere}

Despite all efforts, it is not clear whether the observed oscillations are the response forced by global $p$-modes, eigenmodes of the sunspot itself, or quiet Sun acoustic waves transformed into magneto-acoustic waves in the magnetized atmosphere of a sunspot. In addition, it is hard to distinguish whether the observed magnetic field oscillations are intrinsic oscillations produced by some type of wave propagating in a sunspot, or whether they are produced by instrumental or time-dependent opacity effects $[8,55,72]$. The assumption that time variations of the opacity of the solar atmosphere can cause the observed magnetic field oscillations was made by Lites et al. [55] soon after the detection of these oscillations. All observations using spectral lines as a source of information are influenced by this effect, even if the magnetic field vector is recovered by inversion methods. In the latter case it seems to be the only distorting factor since the influence of other atmospheric parameters is carefully excluded.

The effect consists of the following. The absolute value of the magnetic field strength in the sunspot umbra in photosphere decreases with height by approximately 2-3 G per $\mathrm{km}$ (see, for example, [85]). Any compressible wave (accompanied by oscillations of pressure and density) will imply time variations of the continuum absorption coefficient. The latter will result in the displacements of the line-forming region. Observations in this line will give values of the magnetic field strength corresponding to lower or higher layers in the atmosphere, leading (in the case of the magnetic field gradient) to larger or lower values of the magnetic field strength.

Although exact calculations have not been performed yet, Rüedi et al. [72] have confirmed the assumptions of Lites et al. [55] about the importance of this effect by means of the numerical modeling. The authors have applied the theoretical model for oscillations of Cally \& Bogdan [19], Cally et al. [20] and synthesized the time sequence of the Ni I $\lambda 676.8 \mathrm{~nm}$ line profiles used by MDI. The theoretical profiles were convolved with the filter functions of MDI in a similar way as is done on board $\mathrm{SOHO}$ and the magnetograms were obtained. The amplitude of oscillations of the magnetic field obtained from the synthesized magnetograms was of $7.2 \mathrm{G}$, while model for oscillations it was just $2.5 \mathrm{G}$ at the heights of formation of the Ni I $\lambda 676.8 \mathrm{~nm}$ line. Rüedi et al. [72] came to the conclusion that a major part of magnetogram signal is not caused by intrinsic oscillations in the magnetic field but rather by oscillations in density and temperature. This effect arises in MDI observations even in the absence of the magnetic field gradient, owing to the intensity oscillations in the Ni I $\lambda 676.8$ line. The phase shifts between the velocity and magnetic field oscillations in the case of zero gradient has an opposite sign to that observed by MDI. If the magnetic field has a gradient, the phase of the magnetic field oscillations depends on its value. At the gradient of $3 \mathrm{G} \mathrm{km}^{-1}$ it differs by 180 degrees from the zero gradient case. Both the amplitude and the phase of the magnetic field oscillations due to opacity effects obtained in the numerical experiment of Rüedi et al. [72] are similar to those registered in MDI observations. Thus, the vertical gradient of the magnetic field in sunspots plays an important role and can define the observed properties of oscillations.

\section{Theoretical models}

Theoretical modeling of oscillations in the magnetized atmosphere has achieved a significant level of sophistication. A number of models are developed for the different magnetic field configuration, various boundary conditions, a homogeneous or stratified atmosphere, etc. Here we shall briefly consider only those models that were used most successfully for the interpretation of oscillations in sunspots. The models can be virtually classified into two main classes - analytical and numerical.

\section{Analytical models}

The analytical solution of the system of the magnetohydrodynamical (MHD) equations for the case of a vertical (parallel to the gravity direction) constant magnetic field is known since the 1970s [86]. The method of seeking the solution was suggested for the first time by Ferraro \& Plumpton [26]. An example of the system of the linearized MHD equations is given below:

$$
\begin{gathered}
\frac{\partial \rho_{1}}{\partial t}+\mathbf{v}_{1} \nabla \rho_{0}+\rho_{0} \nabla \mathbf{v}_{1}=0 \\
\rho_{0} \frac{\partial \mathbf{v}_{1}}{\partial t}+\nabla P_{1}-\mathbf{g} \rho_{1}+\frac{1}{4 \pi} \nabla\left(\mathbf{B}_{0} \mathbf{B}_{1}\right)-\left(\mathbf{B}_{0} \nabla\right) \mathbf{B}_{1}=0 \\
\rho_{0} c_{v} \frac{\partial T_{1}}{\partial t}+P_{0}\left(\nabla \mathbf{v}_{1}\right)=-\frac{1}{\tau_{R}} \rho_{0} c_{v} T_{1} \\
\frac{\partial \mathbf{B}_{1}}{\partial t}-\left(\mathbf{B}_{0} \nabla\right) \mathbf{v}_{1}+\mathbf{B}_{0}\left(\nabla \mathbf{v}_{1}\right)=0
\end{gathered}
$$


where the variables have the usual meaning. Here, the equation of energy conservation (5) contains on the righthand side the term describing energy losses due to the radiative relaxation under the Newtonian cooling approximation. Assuming the time and horizontal dependence of all first-order variables of the form $\exp i\left(\omega t+k_{x} x\right)$, the system $(3-6)$ is reduced to fourth-order differential equations for a variable $\xi=\omega H / V_{a}$, where $\omega$ is the frequency of oscillations, $H$ is the scale height of pressure of the isothermal atmosphere, and $V_{a}$ is the Alfven speed. The first two quantities are constants in the model while $V_{a}=B_{0} / \sqrt{4 \pi \rho}$ depends on height according to the exponential fall in density at $B_{0}=$ const. The solutions of the differential equation for $\xi$ can be expressed in terms of Meijer $G_{24}^{12}$ functions, which are determined as complex contour integrals.

The theory of oscillations in the isothermal magnetized atmosphere was then advanced in works by Zhugzhda \& Dzhalilov [89-91], Leroy \& Schwartz [53], Babaev et al. [6,7], and Cally [18]. It was generalized for the case of a constant inclined magnetic field by Zhugzhda \& Dzhalilov [89-91], and for the case of non-adiabatic oscillations by Babaev et al. [6,7]. The solutions of the system are magneto-acoustic-gravity waves in a stratified atmosphere, and their properties depend mainly on the ratio between magnetic and gas pressure (i. e. plasma parameter $\beta$ ) and the inclination of the magnetic field.

Babaev et al. [6, 7], Zhugzhda \& Dzhalilov [89-91] analyzed the properties of the solutions in the case of a weak and strong magnetic field and the transformation of waves at the transition from one regime to another. In the case of a weak magnetic field $(\xi \gg 1)$, the asymptotic solutions have the form [90]:

$$
\begin{aligned}
v_{\perp} & =C_{1} \exp \left[-\frac{z}{2 H}+i\left(\omega t+k_{x} x+k_{z} z\right)\right] \\
& +C_{2} \exp \left[-\frac{z}{4 H}+i\left(\omega t+k_{x}(x-\sigma z) \pm \frac{2 \omega H}{V_{a z}}\right)\right]
\end{aligned}
$$

where $v_{\perp}$ is the velocity component perpendicular to the magnetic field, $C_{1}$ and $C_{2}$ are constants, $V_{a z}$ is vertical component of Alfven speed, parameter $\sigma=B_{0 x} / B_{0 z}$ depends on the inclination of the magnetic field, and $k_{z}$ is defined from the relation:

$$
k_{z}^{2}=\frac{\omega^{2}}{c_{s}^{2}} \frac{\left(1+\sigma^{2}\right)}{\gamma}-\frac{1}{4 H^{2}}-k_{x}^{2}\left(1-\frac{(\gamma-1)}{\gamma} \frac{c_{s}^{2}}{\omega^{2} H^{2}}\right) .
$$

Solution (7) defines the two independent modes of oscillation and two complex conjugate solutions can be added to it. In case of a vertical magnetic field $\sigma$ it is equal to zero and $k_{z}$ coincides with the wave vector for the acoustic-gravity waves in the non-magnetized atmosphere. This means that the mode of oscillations with $C_{1}$ in the weak magnetic field represents to first order a usual acoustic-gravity wave. The solution with $C_{2}$ de- scribes a slow magneto-acoustic wave in the weak magnetic field. In the case $V_{a z} \ll \omega / \sigma k_{x}$ this wave propagates with Alfven speed in the direction of $Z$ axis. The given inequality is fulfilled (among other cases) for small $k_{x}$ and $\sigma$, i. e. vertical oscillations in the vertical magnetic field. Thus, the mode with $C_{2}$ in case of the weak magnetic field is similar to the Alfven wave in the non-stratified atmosphere except that its amplitude grows with height as $\exp (-z / 4 H)$.

In case of a strong magnetic field $(\xi \ll 1)$ the asymptotic solution looks like [90]:

$$
\begin{aligned}
v_{\perp} & =C_{1} \exp \left[-\frac{z}{2 H}+i\left(\omega t \pm k_{x} x \pm k_{z} z\right)\right] \\
& +C_{2} \exp \left[ \pm k_{x} z+i\left(\omega t \pm k_{x} x\right)\right]
\end{aligned}
$$

where

$$
k_{z}= \pm \sqrt{\frac{\omega^{2}}{c_{s}^{2}} \frac{\left(1+\sigma^{2}\right)}{\gamma}-\frac{1}{4 H^{2}}}-\frac{2 \sigma k_{x}}{2 H} .
$$

As the value of the wave vector $k_{z}$ depends on the sign of $k_{x}$, the item with $C_{1}$ includes four linearly independent solutions for a slow magneto-acoustic mode in a strong field. In other words, the character of the wave propagation depends on whether $k_{x}$ and $B_{0 x}$ have the same sign. The $C_{2}$ solution corresponds to the fast magnetoacoustic mode. According to Zhugzhda \& Dzhalilov [90] this mode does not propagate in the vertical direction due to a complete reflection but propagates horizontally irrespective of the magnetic field inclination.

In case of an intermediate value of the magnetic field $(\xi \sim 1)$ the solutions of the system of MHD equations cannot be classified as independent modes of oscillations in the sense that they actively interact with each other. The maximum of the interaction between various types of modes and their transformation from one type to another occurs in the region where $\xi \sim 1$ [91]. The computation of the solutions in terms of Meijer functions in the case $\xi \sim 1$ (which is equivalent to $\beta \sim 1$ ) encounters numerical difficulties since the integral (or the series) does not always converge. The observed photospheric 5minute oscillations correspond to the heights of $0-300$ $\mathrm{km}$, depending on a spectral line. In these relatively deep layers $\beta \leq 1$ and the use of the exact solution of the system of MHD equations in terms of Meijer functions for interpretation of oscillations is complicated.

\section{E. Numerical models}

Since the complexity of the MHD equation describing the problem very often makes an analytical solution impossible, recourse is had to numerical approaches. One of the most successful numerical models is that of Bogdan \& Knölker [15], Cally \& Bogdan [19], and Cally et al. [20] who explored a possibility of the existence the of trapped modes of oscillations in the sunspot atmosphere. 
The model atmosphere consists of three layers: the photosphere and chromosphere are represented by MACKKL, the M sunspot model [56], a polytrope below, and an isothermal corona above. The vertical magnetic field is constant in all the layers. The initial system of MHD equations is the same as in the paper by Zhugzhda \& Dzhalilov [90] (see Eqs. 3-6 above), the perturbations are assumed to be adiabatic. A numerical solution of the system of MHD equations, depending on the parameter, leads to a discrete set of damped modes for a prescribed real horizontal wavenumber [19] or to a set of evanescent spatial modes for the prescribed real frequencies $\omega[20]$. In the first case [19] in the weak magnetic field the imaginary part of complex eigenfrequencies reduces to zero, i. e. the values of frequencies tend to real values for the polytropic non-magnetized atmospheres. In the strong magnetic field, the damping of oscillations decreases with increasing horizontal wave number. At some values of the wave number the imaginary part of frequency becomes equal to zero which corresponds to a trapped mode of oscillations in a sunspot. According to the estimates of Cally \& Bogdan [19] the eigenperiods of oscillations of sunspots appear to be in the 3 -minute period band. In the case that the free parameters of the model are the real frequencies of oscillations, imaginary values of horizontal wave number (according to [20]) mean the damping to oscillations in the direction from the penumbra towards the center of a sunspot.

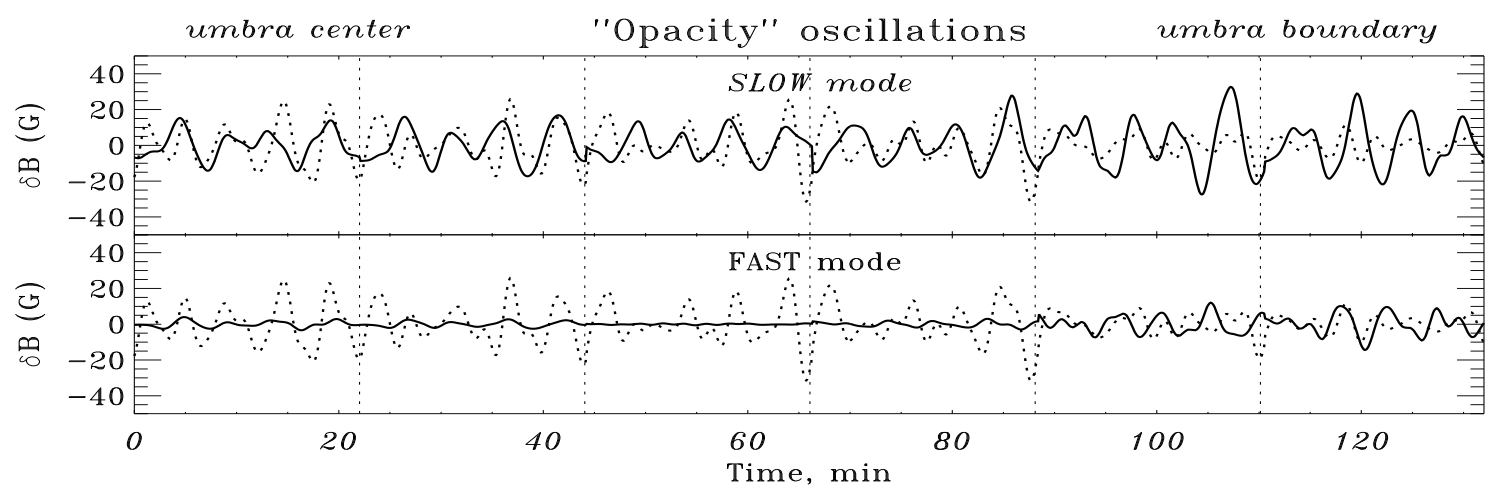

Intrinsic oscillations

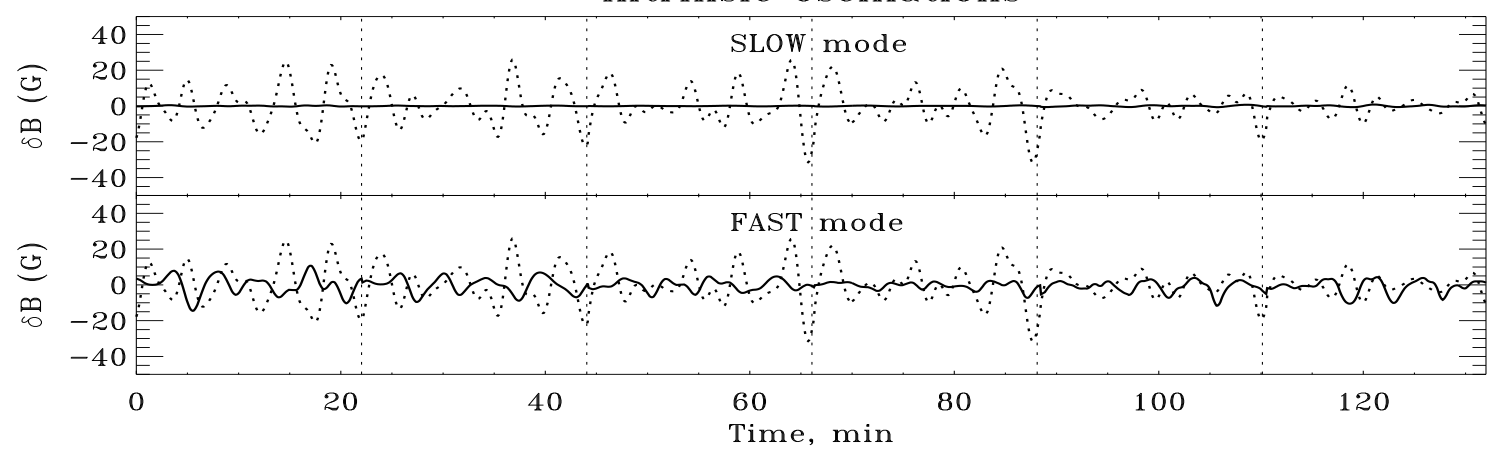

The fit

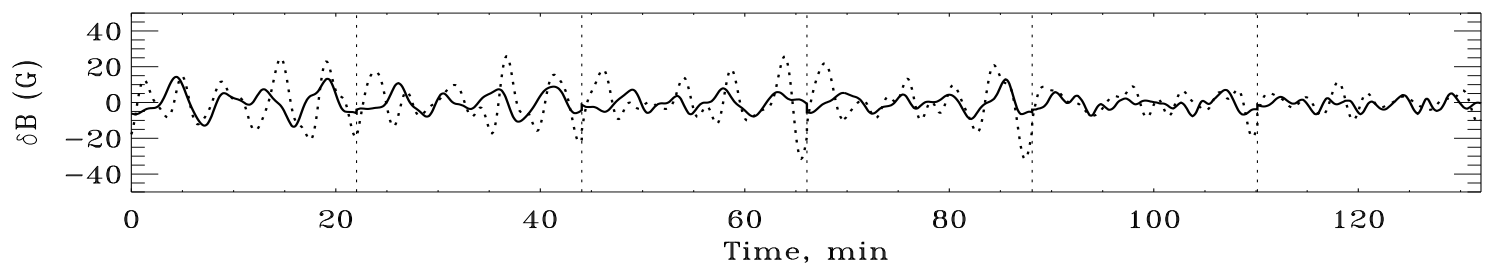

Fig. 6. Top panel: Dotted curves - observed variations of magnetic field strength at $\log \tau_{5000}=-1$. The oscillations are filtered in the frequency range $2-8 \mathrm{mHz}$. Solid curves - variations of magnetic field produced by temporal variations of opacity due to the SLOW (upper plot) and FAST (lower plot) magneto-acoustic modes. Individual 22-min time series from six adjacent pixels are put together to make the oscillations more visible. The farther from the left, the larger the distance to the umbra center. Middle panel: The same for intrinsic variations of magnetic field. Bottom panel: Dotted curve - observations. Solid line - least squares fit to observations from the "opacity" and intrinsic parts of $\delta B$ variations of both modes (see Khomenko et al. [43] for details). 
The amplitudes of oscillations of the magnetic field obtained in the numerical model of Cally et al. $[19,20]$ are low, approximately several G, and the phases do not agree with observations $[55,72]$. A serious limitation of the model is the assumption of constant with height vertical magnetic field. As mentioned before, the magnetic field inclination and gradient play an important role in the appearance of the observed oscillations and neglecting these agents does not allow an adequate comparison with observations.

There are thus several questions to be answered. Do we observe intrinsic magnetic field oscillations? How important is the effect of temporal variations of opacity? Which types of waves can explain the observed magnetic field variations? To answer the question on the nature of sunspot oscillations it is necessary on the one hand to have precise knowledge of the oscillating parameters and relations between them. On the other hand, theoretical models should reproduce closely the conditions in the solar atmosphere where the oscillations are observed, the configuration of the magnetic field in sunspots and the observational procedure.

Some work in this direction has been done by Khomenko et al. [43]. An attempt was made to account for the observational procedure and for the observed structure of the sunspot as close as possible, even at the cost of some simplification of the wave propagation model. A solution of the MHD wave equation was considered in a stratified atmosphere with a non-constant inclined magnetic field. The magnetic field vector and other parameters of the atmosphere (such as pressure, temperature, etc.) were taken from the inversion of the full Stokes vector of the infrared Fe I lines by Bellot Rubio et al. [8]. The magnetic field gradient was taken into account by the method of slabs, i. e. by dividing the atmosphere into a series of horizontal slabs where the atmosphere parameters change slightly and the solution for the uniform atmosphere can be applied. Despite these serious simplifications the intrinsic magnetic field oscillations and the oscillations due to opacity effects were computed for the two magneto-acoustic modes and compared with observations by Bellot Rubio et al. [8]. The oscillations of the magnetic field due to opacity variations are shown in the upper panel of Fig. 6. The two modes behave quite differently. The slow mode produces significant "opacity" magnetic field oscillations. At the umbra center, the amplitude and phase of this mode looks quite similar to the observational data. At the umbra/penumbra boundary, the amplitude is much larger and the theoretical curve does not describe the observations. The fast mode produces negligible opacity variations in all the models except the last two closest to the umbra border. The behavior of the intrinsic magnetic field oscillations differs strongly from those due to opacity effects (central panel of Fig. 6). The amplitudes of the intrinsic $\delta B$ oscillations produced by the slow mode are extremely low. At the same time the $\delta B$ oscillations of the fast mode are of the same order of magnitude as the observations. The intrinsic $\delta B$ oscillations are in antiphase with the observations, which is in agreement with the results of Rüedi et al. [72].
Comparison with observations revealed that the observed oscillations could be a result of magneto-acoustic modes in sunspot umbrae and that the oscillations can only be reproduced if we assume that two fast and slow modes co-exist in a sunspot, and that their relative contribution into the observed oscillations changes with the distance from sunspot radius. An important conclusion of Khomenko et al. [43] is that modeled and observed oscillations have similar amplitudes.

\section{CONCLUSIONS}

In spite of the vast amount of knowledge gathered about 5-minute oscillations and improvements in theoretical models, many important questions remain unanswered. Some of these questions are listed below.

- What are the amplitudes and phases of oscillations above granules and intergranules? How does the effect depend on the spectral lines used for observations?

- How to separate effectively local and global wave phenomena?

- How do properties of oscillations above granules and lanes depend on height?

- Why do evanescent 5-minute oscillations sometimes show phases proper for running sound waves?

- What can explain the differences in behavior of oscillations above granules and lanes - the existence of discrete acoustic sources and/or the modulation of oscillations by convective structure of the solar atmosphere?

- What are the relations between the excitation mechanism of oscillations and their observed relations with the granular pattern?

- Are there relations between the excitation of oscillations with the solar activity?

- What are the additional mechanisms that drive high-energy low- $l$ oscillations?

- The consistent theory of waves in periodic gravitating solar atmosphere does not exist.

- What will the amplitudes and phases of 5-minute oscillations be in the case of modulation by the atmosphere? Will the difference in amplitudes of oscillations above granules and intergranules grow or decrease with height?

- The wavelengths of acoustic oscillations and granulation are nearly of the same order. What effects will cause the reflection of waves on granular inhomogenities?

- Do we observe intrinsic magnetic field oscillations in sunspots? Precise knowledge concerning the oscillating parameters and relations between them is needed. 
- How important is the effect of temporal variations in the opacity of the solar atmosphere?

- Which types of waves can explain the observed magnetic field variations in sunspots? Theoretical models should reproduce closely the conditions in the solar atmosphere where the oscillations are observed, the configuration of the magnetic field in sunspots, and the observational procedure.

- Is there a resonant cavity in sunspots as in the quiet Sun? Do sunspots possess trapped modes of oscillations?

This work was partially supported by INTAS grant 00-00084.
[1] B. T. Babij, A. D. Altman, Soln. Dannye (in Russian) 5, 103 (1969).

[2] R. I. Kostik, E. V. Khomenko, Astron. Zh. (in Russian) 46, N 12, 925 (2002).

[3] E. V. Khomenko, Kinem. Fiz. Nebes. Tel (in Russian) 18, N6, 559 (2002).

[4] T. E. Abdelatif, B. W. Lites, J. H. Thomas, Astrophys J. 311, 1015 (1986).

[5] M. Asplund, A. Nordlund, R. Trampedach, C. Allende Prieto, R. F. Stein, Astron. Astrophys. 359, 729 (2000).

[6] E. S. Babaev, N. S. Dzhalilov, Y. D. Zhugzhda, Astron. Reports 39, No 2, 202 (1995).

[7] E. S. Babaev, N. S. Dzhalilov, Y. D. Zhugzhda, Astron. Reports 39, No 2, 211 (1995).

[8] L. R. Bellot Rubio, M. Collados, B. Ruiz Cobo,I. Rodríguez Hidalgo, Astrophys J. 534, 989 (2000).

[9] N. J. Balmforth, Mon. Not. R. Astron. Soc. 255, 639 (1992).

[10] H. Balthasar, Magnetic Fields and Oscillations, Vol. 184, 1999, edited by B. Schmieder, A. Hofmann, J. Staude, (ASP Conference Series, Kluwer, Dordrecht), p. 141.

[11] H. Balthasar, Sol. Phys. 187, 389 (1999).

[12] S. Bi, R. Li, Astron. Astrophys. 335, 673 (1998).

[13] L. Bierman, Z. Astrophys. 25, 161 (1948).

[14] T. J. Bogdan, Sol. Phys. 192, 373 (2000).

[15] T. J. Bogdan, M. Knölker, Astrophys J. 339, 579 (1989).

[16] T. M. Brown, Astrophys J. 371, 396 (1991).

[17] T. M. Brown, T. J. Bogdan, B. W. Lites, J. H. Thomas, Astrophys J. 394, L65 (1992).

[18] P. S. Cally, Astrophys J. 548, 473 (2001).

[19] P. S. Cally, T. J. Bogdan, Astrophys J. 402, 721 (1993).

[20] P. S. Cally, T. J. Bogdan, E. G. Zweibel, Astrophys J. 437, 505 (1994).

[21] W. J. Chaplin, Y. Elsworth, R. Howe, G. R. Isaak, C. P. McLeod, B. A. Miller, New, R. Mon. Not. R. Astron. Soc. 287, 51 (1997).

[22] T. L. J. Duvall, S. M. Jefferies, J. W. Harvey, Y. Osaki, M. A. Pomerantz, Astrophys J. 410, 829 (1993).

[23] G. Eriksen, P. Maltby, Astrophys J. 148, 833 (1967).

[24] O. Espagnet, R. Muller, T. Roudier, P. Mein, N. Mein, J. M. Malherbe, Astron. Astrophys. 313, 297 (1996).

[25] J. W. Evans, R. Michard, Astrophys J. 136, 493 (1962).

[26] V. C. A. Ferraro, C. Plumpton, Astrophys J. 127, 459 (1958).

[27] T. Foglizzo, Astron. Astrophys. 339, 261 (1998).

[28] E. N. Frazier, Astrophys J. 152, 557 (1968).

[29] D. Georgobiani, A. G. Kosovichev, R. Nigam, A. Nordlund, R. F. Stein, Astrophys J. 530, 934 (2000).

[30] P. Goldreich, D. A. Keeley, Astrophys J. 211, 934 (1977).
[31] P. Goldreich, P. Kumar, Astrophys J. 326, 462 (1988).

[32] P. Goldreich, P. Kumar, Astrophys J. 363, 694 (1990).

[33] P. Goldreich, N. Murray, P. Kumar, Astrophys J. 424, 466 (1994).

[34] P. R. Goode, D. Gough, A. Kosovichev, Astrophys J. 387, 707 (1992).

[35] P. R. Goode, L. H. Strous, T. R. Rimmele, R. T. Stebbins, Astrophys J. 495, L27 (1998).

[36] A. Hanslmeier, A. Kučera, J. Rýbak, A. Neunteufel, H. Wohl, Astron. Astrophys. 356, 308 (2000).

[37] A. Hanslmeier, A. Kučera, J. Rýbak, H. Wohl, The Dynamic Sun, 2001, edited by A. Hanslmeier, M. Messerotti, A. Veronig, p. 267.

[38] N. M. Hoekzema, P. N. Brandt, R. J. Rutten, Astron. Astrophys. 333, 322 (1998).

[39] N. M. Hoekzema, R. J. Rutten, Astron. Astrophys. 329, 725 (1998).

[40] N. M. Hoekzema, R. J. Rutten, P. N. Brandt, R. A. Shine, Astron. Astrophys. 329, 276 (1998).

[41] T. Horn, J. Staude, V. Landgraf, Sol. Phys. 172, 69 (1997).

[42] E. Khomenko, Helio- and Astroseismology at the Dawn of the Millenium, Vol. 464, 2001, edited by A. Wilson, ESA SP, p. 456.

[43] E. V. Khomenko, M. Collados, L. R. Bellot Rubio, Astrophys J. 588, 606 ( 2003).

[44] E. V. Khomenko, R. I. Kostik, N. G. Shchukina, Astron. Astrophys. 369, 660 (2001).

[45] M. Kiefer, M. Stix, H. Balthasar, Astron. Astrophys. 359, 1175 (2000).

[46] A. G. Kosovichev, Magnetic fields and Oscillations, Vol. 184, Astron. Soc. Pac. Conf. Series., 1999, edited by B. Schmieder, A. Hofmann, J. Staude, p. 151.

[47] A. G. Kosovichev, V. V. Zharkova, Nature 393, 317 (1998).

[48] R. Kostik, N. Shchukina, Astron. Lett. 25, No.10, 678 (1999).

[49] R. Kostik, N. Shchukina, E. Khomenko, Magnetic Fields and Solar Processes, Vol. 448, 9th European Meeting on Solar Physics, ESA-SP, 2000, edited by A. Wilson, p. 319.

[50] R. I. Kostik, T. V. Orlova, Sol. Phys. 36, 279 (1974).

[51] P. Kumar, P. Goldreich, Astrophys J. 342, 558 (1989).

[52] R. B. Leighton, R. W. Noyes, G. W. Simon, Astrophys J. 135, 474 (1962).

[53] B. Leroy, S. J. Schwartz, Astron. Astrophys. 112, 84 (1982).

[54] M. J. Lighthill, Proc. R. Soc. London A 211, 564 (1952).

[55] B. W. Lites, J. H. Thomas, T. J. Bogdan, P. S. Cally, 
Astrophys J. 497, 464 (1998).

[56] P. Maltby, E. H. Avrett, M. Carlsson, O. Kjeldsth-Moe, R. L. Kurucz, R. Loeser, Astrophys J. 306, 284 (1986).

[57] F. Meyer, H. U. Schmidt, Z. Astrophys. 65, 274 (1967).

[58] P. F. Moretti, A. Cacciani, A. Hanslmeierx, A. Messerottix, M. Oliviero, W. Otruba, W. Severinox, A. Warmuth, Astron. Astrophys. 372, 1038 (2001).

[59] K. Murawski, E. N. Penelovski, Astron. Astrophys. 359, 759 (2000).

[60] S. Musman, Sol. Phys. 36, 313 (1974).

[61] R. Nigam, A. G. Kosovichev, Astrophys J. 514, L53 (1999).

[62] R. Nigam, A. G. Kosovichev, P. H. Scherrer, J. Schou, Astrophys J. 495, L115 (1998).

[63] A. Nordlund, R. F. Stein, Astrophys J. 546, 576 (2001).

[64] A. A. Norton, R. K. Ulrich, R. I. Bush, T. D. Tarbell, Astrophys J. 518, L123 (1999).

[65] R. W. Noyes, R. W. Leighton, Astrophys J. 138, 631 (1963).

[66] M. J. Penn, B. J. LaBonte, Astrophys J. 415, 383 (1993).

[67] M. P. Rast, Astrophys J. 534, 462 (1999).

[68] S. R. Restaino, R. T. Stebbins, P. R. Goode, Astrophys J. 408, L57 (1993).

[69] T. R. Rimmele, P. R. Goode, E. Harold, R. T. Stebbins, Astrophys J. 444, L119 (1995).

[70] C. S. Rosenthal, J. Christensen-Dalsgaard, A. Nordlund, R. F. Stien, R. Trampedach, Astron. Astrophys. 351, 689 (1999).

[71] B. Ruiz Cobo, J. C. del Toro Iniesta, Astrophys J. 398, 375 (1992).

[72] I. Rüedi, S. K. Solanki, T. Bogdan, P. Cally, in: K. N. Nagendra, J. O. Stenflo (eds.) Solar Polarization (Kluwer, Dordrecht, 1999), p. 337.
[73] I. Rüedi, S. K. Solanki, J. Stenflo, T. Tarbell, P. H. Scherrer, Astron. Astrophys. 335, L97 (1998).

[74] H. U. Schmidt, J. B. Zirker, Astrophys J. 138, 1310 (1963)

[75] A. Settele, T. A. Carroll, I. Nickelt, A. A. Norton, Astron. Astrophys. 386, 1123 (2002).

[76] S. K. Solanki, Astron. Astrophys. 11, 153 (2003).

[77] J. Staude, in B. Schmieder, A. Hofmann, J. Staude (eds.), Magnetic fields and Oscillations, Vol. 184, 1999, ASP Conference Series (Kluwer, Dordrecht), p. 113.

[78] R. T. Stebbins, P. R. Goode, Sol. Phys. 110, 237 (1987).

[79] R. F. Stein, A. Nordlund, Astrophys J. 546, 585 (2001).

[80] M. Stix, Astron. Astrophys. 4, 189 (1970).

[81] M. Stix, Sol. Phys. 196, 19 (2000).

[82] M. Stix, Y. D. Zhugzhda, Astron. Astrophys. 335, 685 (1998).

[83] L. H. Strous, P. R. Goode, T. R. Rimmele, Astrophys J. 535, 1000 (2000).

[84] R. B. Teplitskaja, Sol. Phys. 6, 18 (1969).

[85] C. Westendorp Plaza, J. C. del Toro Iniesta, B. Ruiz Cobo,V. Martínez Pillet, B. W. Lites, A. Skumanich, Astrophys J. 547, 1130 (2001).

[86] Y. D. Zhugzhda, Astron. Zh 56, 74 (1979).

[87] Y. D. Zhugzhda, Astron. Astrophys. 332, 314 (1998).

[88] Y. D. Zhugzhda, H. Balthasar, Staude, J. Astron. Astrophys. 355, 347 (2000).

[89] Y. D. Zhugzhda, N. S. Dzhalilov, Astron. Astrophys. 112, 16 (1982).

[90] Y. D. Zhugzhda, N. S. Dzhalilov, Astron. Astrophys. 132, 45 (1984).

[91] Y. D. Zhugzhda, N. S. Dzhalilov, Astron. Astrophys. 132, 52 (1984).

\title{
КОЛИВАННЯ В ЛОКАЛЬНИХ СОНЯЧНИХ УТВОРЕННЯХ
}

\author{
Р. Костик, О. Хоменко \\ Головна астрономічна обсерваторія НАН Украӥни, \\ вул. Заболотного, 27, Київ, 03680, Україна
}

\begin{abstract}
Сонце - це єдина зоря, поверхню якої можна спостерігати з такої близької відстані. Просторова роздільна здатність сучасних наземних й орбітальних телескопів досягає 0.1 кутової секунди, що еквівалентно 100 км на сонячному диску. Це дає нові можливості для вивчення тонкої структури сонячної атмосфери: грануляції, локальних коливань, варіяцій магнетного поля у спокійних та активних ділянках. У цій оглядовій статті наведено результати досліджень коливань і їх взаємодії з локальними структурами сонячної атмосфери - грануляцією та сонячними плямами. Розглянуто такі питання: вплив хвиль на контури спектральних ліній, механізми збудження коливань локальними акустичними джерелами, модуляція хвиль конвективною структурою сонячної атмосфери, результати спостереження та математичного моделювання коливань у сонячних плямах. Коротко сформульовано проблеми, які чекають свого розв'язання.
\end{abstract}

